\title{
Deployment and Use of the ATLAS DAQ in the Combined Test Beam
}

S. Gadomski, M. Abolins, I. Alexandrov, A. Amorim, C. Padilla-Aranda, E. Badescu, N. Barros, H. P. Beck, R. Blair, D. Burckhart-Chromek, M. Caprini, M. Ciobotaru, P. Conde-Muino, A. Corso-Radu, B. Di Girolamo, M. Diaz-Gomez, R. Dobinson, M. Dobson, R. Ferrari, M. L. Ferrer, D. Francis, S. Gameiro, B. Gorini, M. Gruwe, S. Haas, C. Haeberli, R. Hauser, R. Hughes-Jones, M. Joos, A. Kazarov, D. Klose, S. Kolos, V. Kotov, G. Lehmann, L. Mapelli, B. Martin, R. McLaren, C. Meirosu, M. Mineev, A. Negri, E. Pasqualucci, V. Perez-Reale, J. Petersen, D. Prigent, Y. Ryabov, C. Sanchez, C. Santamarina-Rios, J. Schlereth, J. E. Sloper, I. Soloviev, S. Stancu, L. Tremblet, G. Unel, W. Vandelli, P. Werner, S. Wheeler, M. Wiesmann, and Y. Yasu

\begin{abstract}
The ATLAS collaboration at CERN operated a combined test beam (CTB) from May until November 2004. The prototype of ATLAS data acquisition system (DAQ) was used to integrate other subsystems into a common CTB setup. Data were collected synchronously from all the ATLAS detectors, which represented nine different detector technologies. Electronics and
\end{abstract}

Manuscript received June 17, 2005.

S. Gadomski is with the Laboratory for High Energy Physics, University of Bern, Switzerland, and also with the Institute of Nuclear Physics, Cracow, Poland (e-mail: szymon.gadomski@cern.ch).

J. Schlereth and R. Blair are with the Argonne National Laboratory, Argonne, IL USA.

C. Haeberli, H. P. Beck, and V. Perez-Reale are with the Laboratory for High Energy Physics, University of Bern, Switzerland.

E. Badescu and M. Caprini are with the Institute of Atomic Physics, National Institute of Physics and Nuclear Engineering, Bucharest, Romania.

C. Meirosu and M. Ciobotaru are with the Faculty of Physics, University of Bucharest.

S. Wheeler is with the University of Alberta, Edmonton, Canada, and also with the University of California, Irvine, CA 92717 USA.

C. Padilla-Aranda, M. Ciobotaru, A. Corso-Radu, B. Di Girolamo, D. Burckhart-Chromek, R. Dobinson, M. Dobson, D. Francis, S. Gameiro, B. Gorini, M. Gruwe, S. Haas, M. Joos, G. Lehmann, L. Mapelli, R. McLaren, B. Martin, C. Meirosu, P. Conde-Muino, J. Petersen, D. Prigent, J. E. Sloper, C. Santamarina-Rios, L. Tremblet, M. Wiesmann, and P. Werner are with the CERN, Geneva, Switzerland.

G. Unel is with the CERN, Geneva, Switzerland, and also with University of California, Irvine, CA 92717 USA

E. Pasqualucci is with the Dipartimento di Fisica, Università di Roma ' $\mathrm{La}$ Sapienza' e I.N.F.N., Rome, Italy.

M. L. Ferrer is with the Laboratori Nazionali di Frascati, INFN, Fracasti, Italy.

A. Kazarov, I. Soloviev, and Y. Ryabov are with Petersburg Nuclear Physics Institute (PNPI), Gatchina, Russia.

S. Stancu is with the University of California, Irvine, CA 92717 USA.

S. Kolos is with Petersburg Nuclear Physics Institute (PNPI), Gatchina, Russia, and also with the University of California, Irvine, CA 92717 USA.

I. Alexandrov, M. Mineev, and V. Kotov with the JINR, Dubna, Russia.

Y. Yasu is with the KEK, High Energy Accelerator Research Organisation, Tsukuba.

A. Amorim, D. Klose, and N. Barros are with the CFNUL/FCUL, Universidade de Lisboa, Portugal.

R. Hughes-Jones is with the Department of Physics and Astronomy, University of Manchester, Manchester, U.K.

M. Abolins and R. Hauser are with the Department of Physics and Astronomy, Michigan State University, East Lansing, MI 48823 USA.

R. Ferrari, A. Negri, and W. Vandelli are with the Dipartimento di Fisica Nucleare e Teorica, Università di Pavia e INFN, Pavia, Italy.

M. D. Gomez is with the Section de Physique, Université de Genève, Geneva, Switzerland.

C. Sanchez is with the Universidad Autonoma de Barcelona, Barcelona, Spain.

Color versions of Figs. 1 and 2 are available online at http://ieeexplore.ieee. org.

Digital Object Identifier 10.1109/TNS.2006.878290 software of the first level trigger were used to trigger the setup. Event selection algorithms of the high level trigger were integrated with the system and were tested with real detector data. The possibility to operate a remote Event Filter farm synchronized with the ATLAS Trigger and Data Acquisition System (TDAQ) was also tested. Event data, as well as detector conditions data, were made available for offline analysis.

\section{INTRODUCTION}

$\mathbf{T}$ THE ATLAS experiment at the Large Hadron Collider at CERN is expected to start taking data in 2007. The experiment is described in another article in these proceedings [1]. In this paper, we first introduce the experiment's Trigger and Data Acquisition system (TDAQ). We then present the combined test beam and discuss the integration of various components of the ATLAS detector, Trigger, and DAQ that was accomplished during the CTB.

\section{THE ATLAS TRIGGER AND DAQ}

The ATLAS TDAQ system is described in detail in [2]. The Trigger has the challenging task of reducing the event rate by a factor of about 200000 while preserving maximum possible acceptance of the interesting physics events. The input event rate is equal to the bunch crossing rate of the LHC machine, which is $40 \mathrm{MHz}$. At this rate, the event data is stored in the pipelines of the front-end readout electronics of all the detectors. While the data are in the pipelines the First Level Trigger (LVL1) rejects or accepts events with a fixed latency of $2.2 \mu \mathrm{s}$. The LVL1 decision is based on calorimeter information (local or global sums of energy in the electromagnetic or hadronic calorimeters), or on the information from special trigger chambers of the muon system. The LVL1 system of ATLAS is implemented in hardware, using custom-built electronics. The maximum accept rate of the LVL1 system is $75 \mathrm{kHz}$, with an upgrade possibility to $100 \mathrm{kHz}$.

Data of events accepted by the LVL1 are sent from the front-end electronics of the detectors into the off-detector electronics, the Readout Drivers (ROD) [3]. From the RODs, which are detector-specific, the data are sent into Readout Buffers (ROB) [4], which are custom-built cards mounted in PCs of the DAQ system. The data is sent using the S-LINK technology [5], which can carry up to $160 \mathrm{MB} / \mathrm{s}$. All ROBs are identical. 


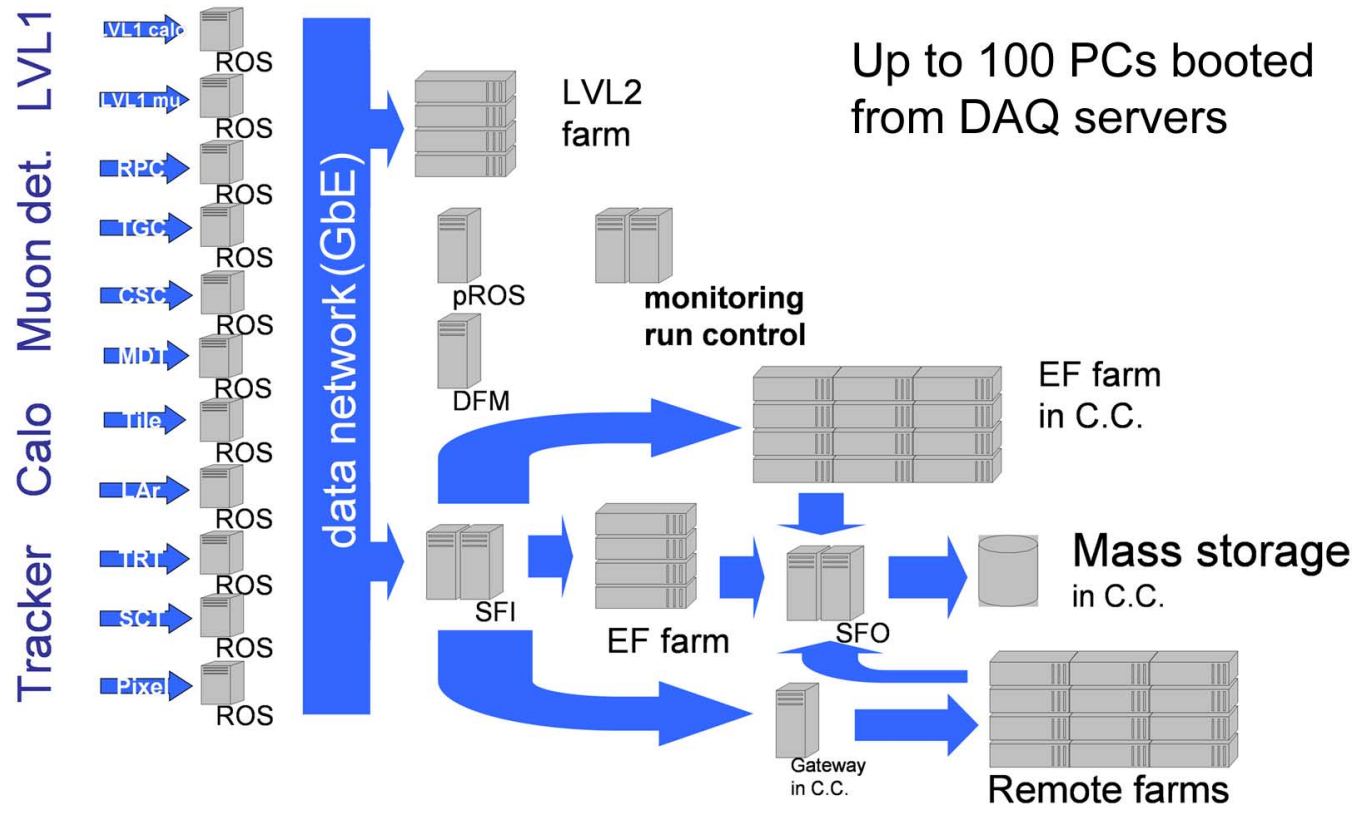

Fig. 1. Schematic diagram illustrating the flow of event data in the Combined Test Beam setup. Most of the components were installed in counting rooms close to the beam line in CERN North Area. The components labeled "in C.C" were in the Computer Centre of CERN. The remote Event Filter farm was in Cracow. The arrows on the left hand side represent data input from prototypes of various ATLAS detectors.

From this point onwards the data stays in the realm of Linux PCs connected with an Ethernet network.

While the data is in the ROBs the Second Level Trigger (LVL2), which will be implemented using event selection algorithms running on PCs, accepts or rejects the events with an average latency of around $10 \mathrm{~ms}$. The LVL2 selection is guided by the regions of interest, which are defined by the LVL1 decision. The LVL2 can reject a large fraction of unwanted events that trigger at LVL1 accessing the data from a small fraction of the ROBs, of the order of $1 \%$ to $2 \%$ on average. An event selection algorithm has the freedom to define which ROBs it needs to access to make the decision. The maximum output rate of the LVL2 is about $3 \mathrm{kHz}$.

Events accepted by the LVL2 are built, i.e., the data from all ROBs is assembled together. The complete events are sent to the third level of event selection done in the Event Filter. The Event Filter will be a large farm of PCs where an event can take on average around one second of CPU time to be processed. After this final step of online selection the event rate is reduced to around $200 \mathrm{~Hz}$ and the data is stored for offline processing.

\section{COMBINED TeSt BeAm OF ATLAS IN 2004}

The ATLAS collaboration has organized beam tests of detector prototypes in one beam line, located in the North Area at CERN Prevessin, for several years. During many of the tests detectors were operated in combined setups. The CTB of 2004 went much beyond that. The objective was to integrate all the ATLAS detectors, as well as all the components of the Trigger and DAQ, in one working system taking data in the beam. The exercise had several objectives:

- exercise the integration of all the systems;

- gain experience with their combined operation;

— study detector response to different particles;

— try particle identification by combining information from different detectors;
— validate the simulation;

- develop and test components of the offline analysis software.

In order to reach those objectives it was important that either the actual components of ATLAS or their final prototypes were used.

\section{INTEGRATION OF SUbSYSTEMS WiTH THE ATLAS DAQ}

The DAQ system of ATLAS was used as the core component of the CTB setup. The gradual buildup of the setup was done by integrating other systems with the DAQ. This concerned all the detectors (and their detector-specific parts of the DAQ) as well as all the components of the Trigger system. The DAQ also provided all the necessary links to the offline software, as discussed later in this section.

The DAQ is implemented mostly in software. The DAQ applications run on commercial processors (Linux PCs) connected with Ethernet network. The DAQ consists of two major subsystems.

1) The Data Flow provides all the applications responsible for the movement of data.

2) The Online Software is providing Run Control, Configuration Database, Monitoring and an Interactive Graphical User Interface (IGUI). The services are used by the Data Flow as well as by the detector-specific DAQ and by the Trigger.

In the following we discuss the integration of the DAQ with other parts of the CTB setup. A schematic diagram of the combined setup is shown in Fig. 1.

\section{A. Integration of Detectors With the DAQ}

The integration of a detector with the DAQ system happens at the level of a ROD Crate. RODs are detector-specific VME modules that control the detector front-end readout electronics. 
The RODs also receive detector data. Each ROD crate has a controller, which is a single board computer running Linux. On the controller one can run ROD Crate DAQ software. This software is built on a common skeleton provided by the DAQ and contains "plugins" that are detector-specific. ROD Crate DAQ assures integration with the Run Control of the DAQ (i.e., an appropriate response to commands coming from the central DAQ console, such as 'start run'). The ROD Crate DAQ also takes care of detector-specific configuration and monitoring.

For monitoring and other diagnostic purposes the ROD Crate DAQ may send detector data to an Ethernet network connected to the crate controller. However during normal running the data is sent directly from the ROD modules, via dedicated readout links, to ROBs mounted in Readout System PCs (ROS). The ROS PCs are physically connected to particular ROD modules, but they are otherwise not detector-specific. They belong to the common DAQ.

A detector is integrated with the DAQ when the ROD Crate DAQ responds to the Run Control commands and the event data flows into the corresponding ROS. In beam test this was done for nine different subdetectors of ATLAS: three subsystems of the Inner Tracker, two calorimeters and four types of muon chambers.

\section{B. Integration of the First Level Trigger With the DAQ}

The First Level Trigger has three major parts: the calorimeter LVL1, the muon LVL1, and the Central Trigger Processor (CTP). From the DAQ point of view, the integration with the LVL1 system resembles what is done to integrate detectors. The steps as described above need to be accomplished for the three parts. In addition the decision of the LVL1 can be used to trigger all the detectors participating in the beam test. In the Combined Test Beam, the full chain was assembled and tested:

- information from real detectors was used to drive the logic of the calorimeter LVL1 and of the muon LVL1;

— information from calorimeter and muon LVL1 subsystems was transferred to the CTP;

- decision of the CTP was used to trigger up to nine other ATLAS detectors installed in the beam test.

The full chain was tested with success. The results concerning the CTP are presented in [6].

\section{Integration of the High Level Trigger With the DAQ}

Both components of the ATLAS High Level Trigger, LVL2 and Event Filter, were integrated with the DAQ in the Combined Test Beam. This integration had several aspects:

- control of the processes;

- passing of the Region of Interest information;

- flow of event data to HLT processors;

— integration of the algorithms.

All the processes of the HLT were started using Online Software Run Control infrastructure and were controlled from the central run control IGUI. A custom hardware component named Region of Interest Builder, which is an interface between the LVL1 and the LVL2, was tested and integrated with the beam test setup.

The flow of event data was assured by components of Data Flow software. For the LVL2 the application named Second
Level Trigger Processing Unit (L2PU) fetched data from the ROS PC holding ROB buffers. The Event Filter Dataflow process was receiving data from Sub-Farm Interface processes, which were doing event building including all the ROBs. Following the Event Filter decision the events were sent to another process, named Sub-Farm Output, which was saving the data for offline processing.

The event selection algorithms, which are developed in the ATLAS offline analysis framework ATHENA, were also integrated. In order to be part of LVL2 the algorithms need to run in the L2PU application. The libraries representing the code of the algorithms were linked with the L2PU and the code was executed on event data, coming in real time from the detectors in the test beam.

For the Event Filter the technical mechanism of the integration is different. Separate processes named Processing Tasks (PT) are started on the Event Filter PCs. The Event Filter event selection algorithms are running as part of the PTs in the ATHENA environment. This way the Event Filter algorithms were also exposed to the data arriving in real time from all the ATLAS detectors in the beam. The information from all the detectors, ranging from silicon pixels of the Inner Tracker through calorimeters to the muon chambers, was present inside each event, made available to the algorithm code. The system was configured in such a way that not only the accepted events, but also the rejected ones were saved for further studies.

A possibility of operating a remote Event Filter farm, located outside CERN, was studied in its data flow aspect using the CTB setup. An Event Filter process running in Cracow was receiving a fraction of events, containing detector data, in real time and was sending the data back (without processing it). Similar exercises with other remote Event Filter farms, located in Edmonton, Manchester, and Copenhagen were also done during the CTB, although no real detector data was involved in those cases.

The integration of the High Level Trigger including beam test results is described in more detail in [7] and the concept of remote Event Filter farms is presented in [8].

\section{Online Monitoring of Event Data}

The monitoring infrastructure of the DAQ enabled sampling of event data in all the applications through which the data is flowing, whether in the Data Flow domain or in the Trigger. The event data was then shipped to monitoring clients, where it was analyzed and where the monitoring plots were produced. Algorithms monitoring the quality of event data were also implemented in the Event Filter. The histograms could be displayed online, while the system was taking data, using monitoring clients. One development using the monitoring infrastructure, which was present in the CTB, is described in [13].

\section{E. Connection to Offline Data Analysis}

The event data was saved in sequences of disk files using the event format and the file format that will be used for ATLAS. The data was then moved to a mass storage system at CERN. The offline software of ATLAS was adapted to read the raw event data and to convert it to objects used for further processing and for the analysis. 
The nonevent data, such as detector status and settings, was transferred to a prototype of the Conditions Database. The database was made accessible to the offline analysis algorithms running in ATHENA.

Run bookkeeping information, for instance beam energy or type of run, was saved in the Conditions Database as well. Subsequently the bookkeeping information was moved to the ATLAS Metadata Interface (AMI). This database service is used to keep track of ATLAS data (until the CTB only simulated data) for processing by offline analysis software running on the GRID.

The Combined Test Beam allowed an exercise of all the interfaces between the online and offline: mass storage of the event data, Conditions Database, and the catalog of data sets.

\section{OPERATION OF DAQ IN THE COMBINED TeSt BEAM}

The integration of all the mentioned components into a fully functioning Combined Test Beam system was a gradual process. The detectors and the parts of the trigger were added to the system one after another. As the DAQ was used to integrate all the parts, it was essential that the DAQ itself was a reliable and dependable service. It needed to be available and working nearly every day throughout the CTB period, including preparations before the beam time.

The DAQ system of the Combined Test Beam was supported continuously, seven days a week and 24 hours a day, for about seven months. In order to cope with this demand, without overloading of the small group of core DAQ developers, the TDAQ Support Teams were organized, involving 38 people. The CTB activities were supported by four teams taking biweekly shifts. The teams have contributed to spreading of the knowledge about the DAQ in the larger TDAQ community, as well as in ATLAS in general.

\section{Results of The BeAm Test}

A few numbers can be given to illustrate the scale of the project. Up to $60 \mathrm{DAQ}$ controllers were used to provide Run Control commands to all the software of the CTB setup. Up to 100 PCs were network-booted from the DAQ servers in the CTB setup. This number included ROD crate controllers, PCs used for Data Flow and Online Software applications, as well as prototypes of High Level Trigger event processing farms.

This setup, although already large, represented only about $10 \%$ of the scale of ATLAS Data Flow and a much smaller fraction of the expected size of the HLT farms.

During the beam time the test beam setup was taking data at event rates up to $3 \mathrm{kHz}$, which is comparable to the event building rate planned for ATLAS $(\sim 3.5 \mathrm{kHz})$. The event sizes ranged from a few $\mathrm{kB}$ to about $60 \mathrm{kB}$, depending on the configuration of the setup, which is much smaller then the event size expected for ATLAS. We estimate that maximum peak data rate was of the order of $100 \mathrm{MB} / \mathrm{s}$. Relative to ATLAS requirements (LVL2 input $\sim 3 \mathrm{~GB} / \mathrm{s}$, EF input $\sim 6 \mathrm{~GB} / \mathrm{s}$, EF output $\sim 300 \mathrm{MB} / \mathrm{s}$ ) the data rate of the CTB was small. For this reason the beam test was not seen as a stress-test of Data Flow components. Performance of the Data Flow system is measured using dedicated test beds, as described in [9].
On the other hand the CTB setup represented well the diversity of technologies and systems that will need to be operated together in ATLAS. In order to illustrate this point we can consider a number of subsystem-specific panels, which the users can add to the standard panels of the Interactive Graphical User Interface (IGUI) of ATLAS TDAQ. In the CTB up to 12 such additional panels were added and operated by the users.

The state machine of the TDAQ was exercised, including for the first time all the detector and HLT components. The state transition times were measured and found to be in the range of 30 to $90 \mathrm{~s}$. The longer times have indicated areas where improvements of the TDAQ software are necessary.

The CTB has enabled several other tests and measurements. The results are presented in separate articles and therefore will not be quoted here. In this paper, we shall only briefly introduce the areas in which the measurements were made. For the LVL1, response of trigger electronics to real particles was measured [10], [11]. The latency of the Central Trigger Processor was also measured [6] and a use of data base in real life conditions was tested [12].

Performance and timing of High Level Trigger algorithms was measured [7]. For the Remote Event Filter Farms network bandwidth and latency were measured and the ability to transfer data using Data Flow components was verified [8]. A monitoring system proposed for ATLAS was stress-tested, handling up to 1000 histograms [13]. Performance of the off-detector readout electronics was measured for several detectors, as presented in case of the Liquid Argon Calorimeter in [1].

The total data sample collected by the CTB was about 3 TB. The data of the CTB is still being analyzed at the time when this article is given its final edit, 1.5 years after the end of the beam tests. The colleagues working on detectors profit from the data to study detector response and particle identification, to validate the simulation and to develop the algorithms of the offline software.

\section{OTHER BENEFITS OF THE CTB FOR FURTHER DEVELOPMENT}

It was expected that some missing or inadequate functionality will be discovered in the TDAQ system during the CTB. Such "lessons" were many. Describing them would in most cases require a level of technical detail beyond the scope of this brief article. We shall give here only one example, relevant to the DAQ Configuration Database, which is used by all the systems, including detectors and trigger.

The experience of a smaller beam test setup in 2003 had showed that the configuration was lacking modularity. It was difficult to merge two working DAQ partitions into one combined partition. It was also impossible to disable a part of a system if one did not want to keep it included in a run. While this was not an issue for setups with one or two detector prototypes, it became very inconvenient for setups with several detectors. The problem was analyzed and a solution was implemented before the beam test of 2004 .

Changes were made in the Online Software as well as in the Data Flow software. In 2004 it was noted by many users that modularity of the system was much improved. It was easier to combine different systems. It was also possible to enable or to 


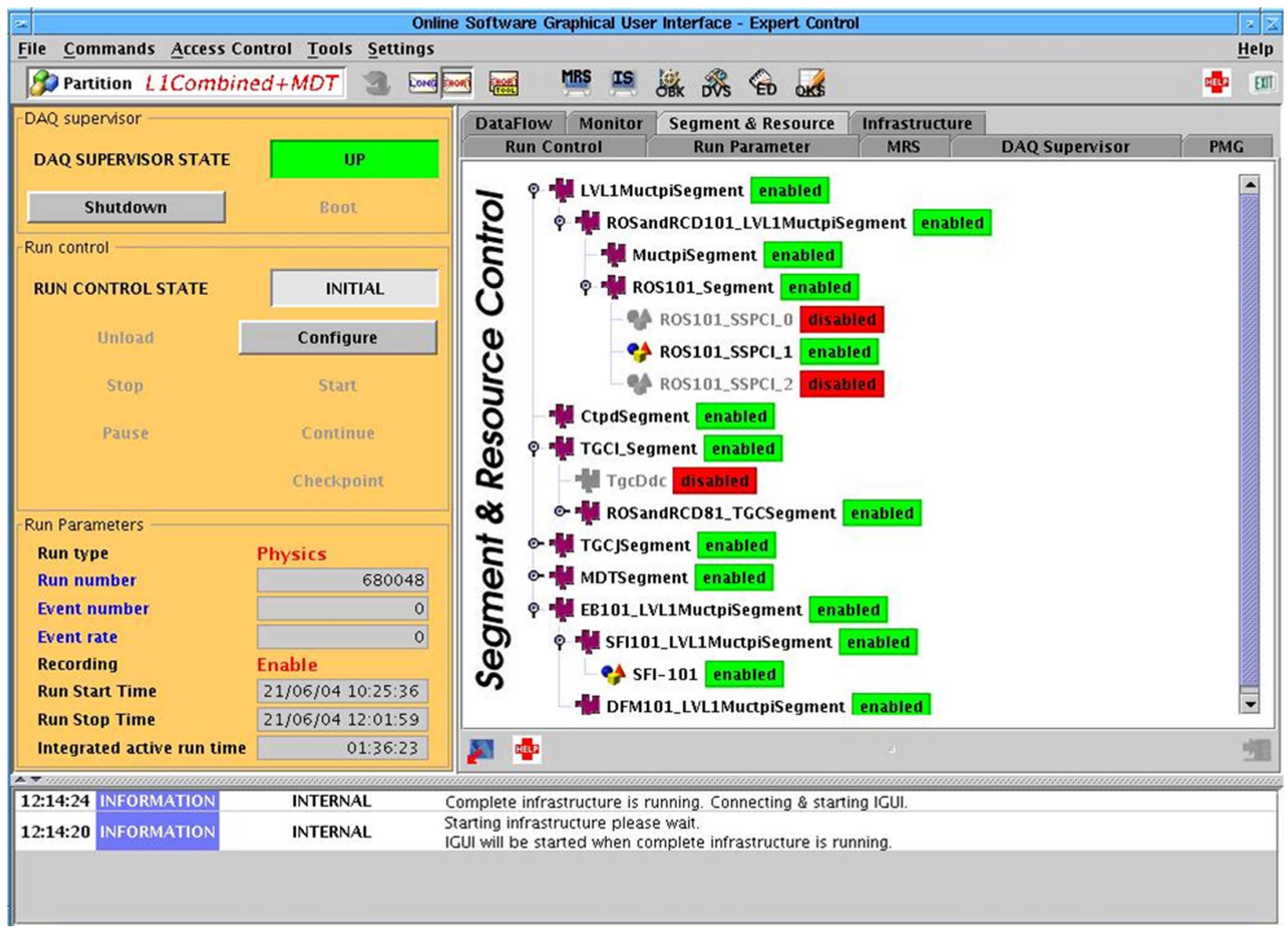

Fig. 2. Interactive Graphical User Interface of the ATLAS DAQ. The panel labeled "Segment and Resource Control" provides the possibility to enable or to disable parts of the system at different scales.

disable parts of the system directly in a panel of Online Software GUI, as shown in Fig. 2. Still some further refinements were required. They have been implemented after the CTB exercise.

One should note that the need of a modular system, although very apparent when working with many detectors, did not manifest itself at all during numerous tests of the TDAQ system, even if done at larger scales than the CTB setup. Without the CTB the issue would not have been discovered before the start of ATLAS commissioning runs combining many detectors. It would have been difficult, probably in fact impossible, to introduce the necessary changes at the stage of advanced commissioning of the experiment.

The educational benefit of the CTB exercise also should not be underestimated. The members of ATLAS involved in the CTB have gained knowledge and experience that will be valuable during the approaching phase of the commissioning.

\section{CONCLUSION}

The Combined Beam Test has given a unique opportunity to bring several subsystems of ATLAS, which are developed separately, into one working setup. The systems that were integrated can be listed as follows:

- ATLAS DAQ (run control, configuration database, monitoring, data flow);
- detector hardware including all electronics and the detector-specific part of the DAQ;

- hardware and software of the LVL1;

- LVL2 and Event Filter (algorithms, software infrastructure; hardware);

— databases for detector conditions and for run bookkeeping;

— offline data analysis software.

Each item in the list breaks down into many components that need to be developed separately. Using them together has given valuable feedback for further development.

The software and the hardware that was used were the latest prototypes for ATLAS and not special developments of the test beam. The DAQ has played the central role in the integration process by providing a reliable and well supported service during seven months.

\section{ACKNOWLEDGMENT}

The authors would like to thank colleagues working on the ATLAS triggers, detectors, and offline for their enthusiasm and hard work during the Combined Test Beam.

\section{REFERENCES}

[1] A. Bazan, F. Bellachia, A. Blondel, J. Colas, M. Citterio, and M. Diaz Gomez et al., "The ATLAS liquid argon calorimeter read out system," IEEE Trans Nucl. Sci, vol. 53, no. 3, pp. 735-740, Jun. 2006. 
[2] ATLAS high level trigger, data acquisition and controls technical design report June 30, 2003, CERN/LHCC/2003-033, ATLAS Collaboration.

[3] [Online]. Available: http://atlas.web.cern.ch/Atlas/GROUPS/ DAQTRIG/DIG/ROD/, Readout driver developments for ATLAS

[4] [Online]. Available: http://www-li5.ti.uni-mannheim.de/research/fpgaprocessors/projects/atlas/, ROBIN development for ATLAS

[5] [Online]. Available: http://cern.ch/s-link/, S-LINK technology

[6] T. Pauly, "The ATLAS level-1 central trigger processor," in Proc. 14th IEEE-NPSS Real Time Conf., Stockholm, Sweden, June 4-10, 2005.

[7] A. dos Anjos, "Deployment of the ATLAS high level trigger," IEEE Trans. Nucl. Sci, vol. 53, no. 4, pp. 2144-2149, Aug. 2006

[8] B. Caron, R. Hughes-Jones, K. Korcyl, C. Meirosu, and J. Langgard Nielsen, "Investigation of the networking performance of remote realtime computing farms for ATLAS trigger DAQ," IEEE Trans. Nucl. Sci., vol. 53, no. 4, pp. 2150-2155, Aug. 2006.

[9] J. Vermeulen, M. Abolins, I. Alexandrov, A. Amorim, A. Dos Anjos, and E. Badescu et al., "ATLAS dataflow: The read-out subsystem, results from trigger and data acquisition system testbed studies and from modelling," IEEE Trans. Nucl. Sci., vol. 53, no. 3, pp. 912-917, Jun. 2006.
[10] R. Achenbach, C. Ay, B. M. Barnett, B. Bauss, A. Belkin, and C. Bohm et al., "Pre-production validation of the ATLAS Level-1 calorimeter trigger system," IEEE Trans. Nucl. Sci., vol. 53, no. 3, pp. 859-863, Jun. 2006.

[11] A. Munar, "Real time data processing of the tile-cal calorimeter of the atlas detector," in Proc. 14th IEEE-NPSS Real Time Conf., Stockholm, Sweden, June 4-10, 2005.

[12] Y. Benhammou, S. Bressler, E. Etzion, D. Lellouch, L. Levinson, and $\mathrm{S}$. Tarem, "The thin gap chambers database experience in test beam and preparations for ATLAS," IEEE Trans. Nucl. Sci., vol. 53, no. 4, pp. 2162-2166, Aug. 2006.

[13] P. F. Zema, "The GNAM monitoring system and the OHP histogram presenter for ATLAS," in Proc. 14th IEEE-NPSS Real Time Conf., Stockholm, Sweden, June 4-10, 2005. 